\title{
The nature of the Cepheid T Antliae
}

\author{
D. G. Turner ${ }^{1}$ and L. N. Berdnikov ${ }^{2}$ \\ 1 Department of Astronomy and Physics, Saint Mary's University, Halifax, Nova Scotia B3H 3C3, Canada \\ 2 Sternberg Astronomical Institute and Isaac Newton Institute of Chile, Moscow Branch, 13 Universitetskij prosp., \\ Moscow 119899, Russia \\ e-mail: berdnik@sai.msu.ru
}

Received 7 April 2003 / Accepted 21 May 2003

\begin{abstract}
The variable star T Antliae, sometimes suspected to be a type II pulsator, is demonstrated to be a classical Cepheid in the third crossing of the instability strip. It exhibits a positive period change of $+0.514 \pm 0.016 \mathrm{~s} \mathrm{yr}^{-1}$, consistent with a star on the blue side of the instability strip and evolving through it for the third time. The Cepheid exhibits no random fluctuations in pulsation period, although superimposed upon its evolutionary $\mathrm{O}-\mathrm{C}$ trend are very subtle variations that may be indicative of orbital motion about an unseen companion. Archival spectroscopic data also indicate that the star sits on the edge of a putative cluster of B-type stars that may be physically associated with the Cepheid. Additional photometric and spectroscopic data for the Cepheid and cluster are needed to strengthen the case. The field reddening of $\mathrm{T}$ Ant established from nearby early-type stars is $E_{B-V}=0.30 \pm 0.01\left(E_{B-V}(\mathrm{~B} 0)=0.316 \pm 0.014\right)$.
\end{abstract}

Key words. stars: distances - stars: variables: Cepheids - Galaxy: open clusters and associations: individual: T Ant

\section{Introduction}

The southern hemisphere variable $\mathrm{T}$ Antliae, with a pulsation period of 5.9 and located at $\alpha_{2000}=09^{\mathrm{h}} 33.85, \delta_{2000}=$ $-36^{\circ} 37^{\prime}, l=264.54, b=+11^{\circ} .12$, is the only Cepheid in the constellation Antlia listed in the fourth edition of the General Catalogue of Variable Stars (Kholopov et al. 1985). Antlia is a rather nondescript constellation of moderately faint stars, one of several such groups in the southern hemisphere invented by Nicolas-Louis de Lacaille (Croswell 2002). It lies just above the Galactic plane along the Puppis-Vela-Carina portion of the southern Milky Way, outside of the region normally included in surveys of disk stars. The relative isolation of T Ant from major groups of early-type stars in nearby Vela and the Cepheid's uncertain catalogue designation as a classical, but possible type II, Cepheid (Harris 1981; Kholopov et al. 1985) help to explain its relative neglect. As we demonstrate here, $\mathrm{T}$ Ant and a number of B-type stars in its immediate vicinity are important objects for future study because there is evidence to indicate that they form a sparse, previously undetected, open cluster in which $\mathrm{T}$ Ant is a likely member. The variable is also identified as a classical Cepheid, as found by Laney (1995) from its BaadeWesselink radius, in the third crossing of the instability strip.

\section{T Antliae}

The literature for T Ant (e.g., Berdnikov 1995, 2002) contains photoelectric observations sufficiently distributed temporally to

Send offprint requests to: $\mathrm{D}$. G. Turner,

e-mail: turner@ap.smu.ca study changes in the star's pulsation period. Such changes are useful for learning about the evolution of Cepheids (Berdnikov 1994; Berdnikov \& Pastukhova 1995; Berdnikov et al. 1997), as well as for detecting orbital motion about a companion or random fluctuations in pulsation period (Berdnikov et al. 2000; Turner et al. 2001; Turner \& Berdnikov 2001). The techniques in current use for such studies have been described by Turner et al. (2001).

Briefly, phased observations of the variable over a restricted number of cycles are used to construct seasonal light curves that are matched to a standard light curve to detect phase shifts indicative of period changes (Hertzsprung's Method). As employed by Berdnikov (1992), the technique makes use of a light curve template constructed from observations. An alternative procedure used by Turner et al. (1999) relies on a set of high-quality observations as a standard, to which independent data sets are matched in magnitude and phase space by least squares techniques. Both algorithms match photometric data for the variable to a reference light curve in the same fashion and produce virtually identical results. They are readily capable of detecting phase shifts for individual data sets to within a precision of \pm 0.001 in phase, which corresponds to uncertainties of a few ten thousandths of a day in O-C data (differences between Observed and Computed times of light maximum).

For $\mathrm{T}$ Ant we followed the method described by Turner et al. (1999) to derive O-C differences for T Ant from a computed linear ephemeris. Care was taken to establish a reference time of light maximum from existing photoelectric observations, the adopted pulsation period being taken from recent 


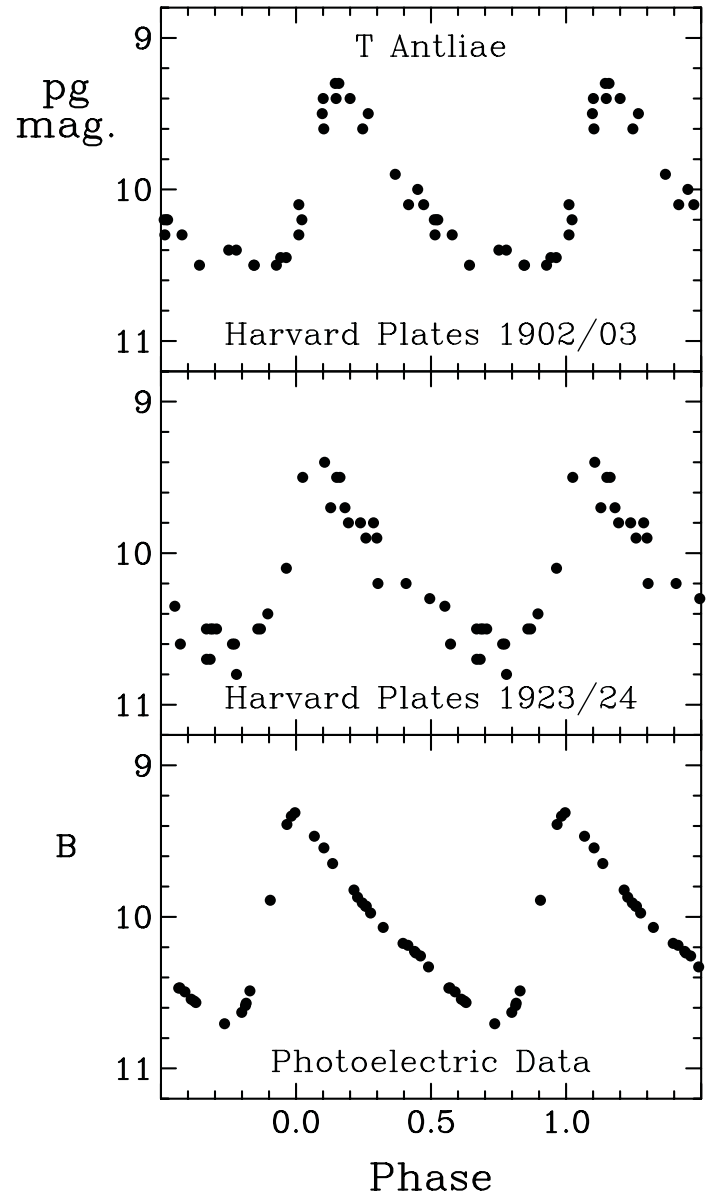

Fig. 1. Photographic light curves for $\mathrm{T}$ Antliae derived from estimates made from Harvard patrol plates for the 1902-03 season (upper) and 1923-24 season (middle), relative to the photoelectric light curve (bottom).

observations of the star by Berdnikov \& Turner (2001). Very precise photometry of T Ant by Pel (1976) was used as a standard for matching data from other epochs in phase and magnitude to the reference set, and the resulting ephemeris was tested with all available photometry for the star to verify its validity over all epochs of observation. The resulting ephemeris for times of light maximum is given by:

$\mathrm{HJD}_{\max }=2436120.608+5.898053 E$,

where $E$ is the number of elapsed cycles.

Magnitudes for $\mathrm{T}$ Ant were also estimated from patrol plates of the field in the Harvard College Observatory Photographic Plate Collection. A reference sequence of six stars surrounding the Cepheid with magnitudes between $B=$ 8.4 and $B=11.0$ was established from broad band data in the Hipparcos/Tycho survey (ESA 1997). The subsequent magnitude estimates for $\mathrm{T}$ Ant had a typical precision of $\pm 0 .{ }^{\mathrm{m}} 1$ to \pm 0.2 , depending on the star's apparent brightness - brighter magnitudes generally being less reliable. A total of 734 estimates of photographic magnitude for $\mathrm{T}$ Ant were derived from plates in the Harvard collection, 699 of them being accurate enough to track the brightness changes of the star in reliable fashion. All data were phased using the ephemeris of Eq. (1).

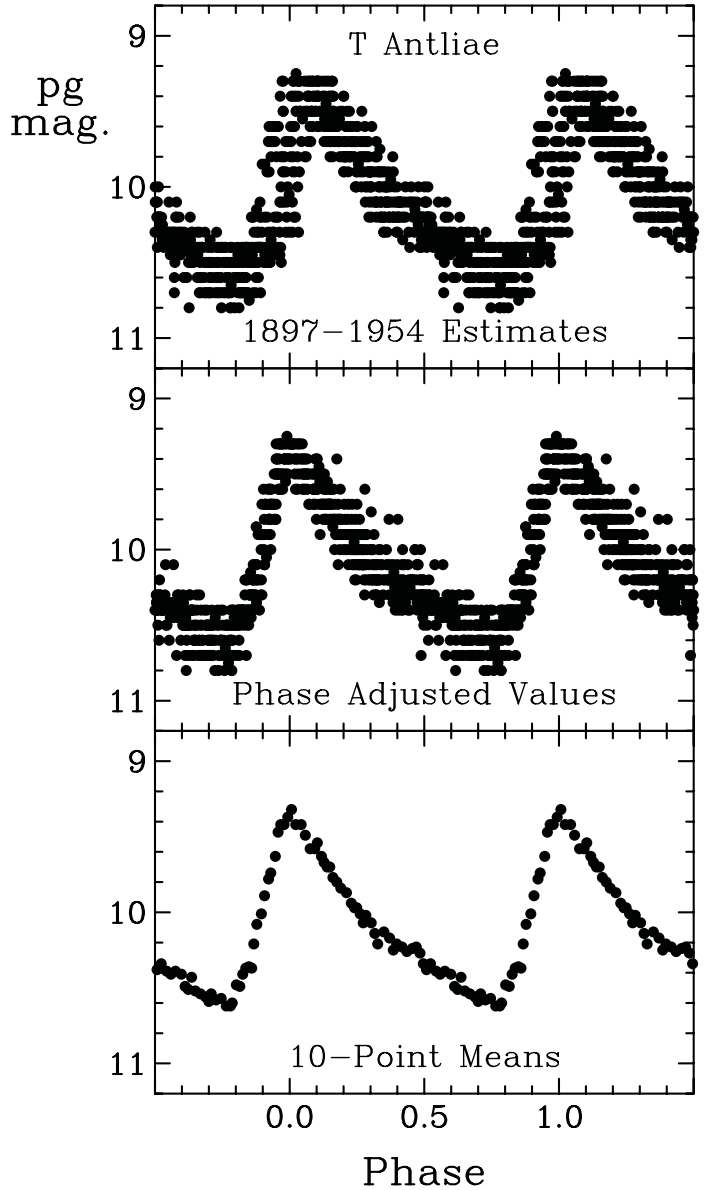

Fig. 2. Magnitude estimates for T Antliae made from Harvard patrol plates covering the 1897 to 1954 interval (upper), as corrected for the Cepheid's gradual period increase (middle), and phase averaged for every ten data points (lower).

An Excel file containing the resulting data is available on request from the authors.

Samples of the derived photographic brightness variations of $\mathrm{T}$ Ant are presented in Fig. 1, which illustrates phased light curves from the 1902/03 and 1923/24 seasons and standard data from the photoelectric photometry of Pel (1976). A good match between photographic plate estimates and photoelectric data is evident in Fig. 1, as are seasonal offsets in phase resulting from period changes in T Ant. Note how well randomly sampled observations of $\mathrm{T}$ Ant from the Harvard patrol plates provide full phase coverage of the star in any particular observing season. There are generally fewer data points for $\mathrm{T}$ Ant during most other seasons sampled by the patrol series, but the exact shape of the light curve was always obvious, even from restricted data samples.

Figure 2 illustrates for reference purposes the full photographic data set for $\mathrm{T}$ Ant along with phase-adjusted data corrected for the gradual change in pulsation period of the star. The bottom section of Fig. 2 illustrates how the photographic light curve can be smoothed by averaging every ten successive data points in pulsation phase. The resulting photographic light curve for T Ant is a good match to the 1976 photoelectric $B$ data for the Cepheid illustrated in Fig. 1. 
Table 1. $\mathrm{O}-\mathrm{C}$ data for $\mathrm{T}$ Antliae.

\begin{tabular}{|c|c|c|c|c|c|c|}
\hline $\mathrm{HJD}_{\max }$ & Cycles, $E$ & $C$ & $\begin{array}{c}\mathrm{O}-\mathrm{C} \\
\text { (days) }\end{array}$ & Weight & $\begin{array}{l}\text { Data Type } \\
\text { (observations) }\end{array}$ & Source \\
\hline 2415165.745 & -3553 & 2415164.826 & +0.924 & 1.0 & $\operatorname{pg}(18)$ & This paper \\
\hline 2416079.895 & -3398 & 2416079.024 & +0.871 & 1.0 & $\operatorname{pg}(29)$ & This paper \\
\hline 2416563.482 & -3316 & 2416562.664 & +0.818 & 1.0 & $\operatorname{pg}(19)$ & This paper \\
\hline 2416887.751 & -3261 & 2416887.057 & +0.694 & 1.0 & $\operatorname{pg}(18)$ & This paper \\
\hline 2417418.653 & -3171 & 2417417.882 & +0.771 & 1.0 & $\operatorname{pg}(23)$ & This paper \\
\hline 2418031.991 & -3067 & 2418031.279 & +0.712 & 1.0 & $\operatorname{pg}(6)$ & This paper \\
\hline 2418409.325 & -3003 & 2418408.755 & +0.570 & 1.0 & $\operatorname{pg}(22)$ & This paper \\
\hline 2418751.489 & -2945 & 2418750.842 & +0.647 & 1.0 & $\operatorname{pg}(17)$ & This paper \\
\hline 2419435.610 & -2829 & 2419435.016 & +0.594 & 1.0 & $\operatorname{pg}(25)$ & This paper \\
\hline 2420096.127 & -2717 & 2420095.598 & +0.529 & 1.0 & $\operatorname{pg}(30)$ & This paper \\
\hline 2420638.730 & -2625 & 2420638.219 & +0.511 & 1.0 & $\operatorname{pg}(25)$ & This paper \\
\hline 2421039.753 & -2557 & 2421039.286 & +0.467 & 1.0 & $\operatorname{pg}(31)$ & This paper \\
\hline 2421370.151 & -2501 & 2421369.577 & +0.574 & 1.0 & $\operatorname{pg}(21)$ & This paper \\
\hline 2421741.631 & -2438 & 2421741.155 & +0.476 & 1.0 & $\operatorname{pg}(26)$ & This paper \\
\hline 2422071.833 & -2382 & 2422071.446 & +0.387 & 1.0 & $\operatorname{pg}(19)$ & This paper \\
\hline 2422449.308 & -2318 & 2422448.921 & +0.387 & 1.0 & $\operatorname{pg}(18)$ & This paper \\
\hline 2423098.073 & -2208 & 2423097.707 & +0.366 & 1.0 & $\operatorname{pg}(10)$ & This paper \\
\hline 2423723.252 & -2102 & 2423722.901 & +0.351 & 1.0 & $\operatorname{pg}(29)$ & This paper \\
\hline 2424472.287 & -1975 & 2424471.953 & +0.334 & 1.0 & $\operatorname{pg}(27)$ & This paper \\
\hline 2425333.562 & -1829 & 2425333.069 & +0.493 & 1.0 & $\operatorname{pg}(24)$ & This paper \\
\hline 2427598.158 & -1445 & 2427597.921 & +0.237 & 1.0 & $\operatorname{pg}(16)$ & This paper \\
\hline 2428276.479 & -1330 & 2428276.198 & +0.281 & 1.0 & $\operatorname{pg}(22)$ & This paper \\
\hline 2428582.994 & -1278 & 2428582.896 & +0.098 & 1.0 & $\operatorname{pg}(11)$ & This paper \\
\hline 2429178.759 & -1177 & 2429178.600 & +0.159 & 1.0 & $\operatorname{pg}(30)$ & This paper \\
\hline 2430057.629 & -1028 & 2430057.410 & +0.219 & 1.0 & $\operatorname{pg}(26)$ & This paper \\
\hline 2430629.675 & -931 & 2430629.521 & +0.154 & 1.0 & $\operatorname{pg}(25)$ & This paper \\
\hline 2431260.758 & -824 & 2431260.612 & +0.146 & 1.0 & $\operatorname{pg}(25)$ & This paper \\
\hline 2431608.806 & -765 & 2431608.597 & +0.209 & 1.0 & $\operatorname{pg}(21)$ & This paper \\
\hline 2431927.259 & -711 & 2431927.092 & +0.167 & 1.0 & $\operatorname{pg}(17)$ & This paper \\
\hline 2432310.670 & -646 & 2432310.466 & +0.204 & 1.0 & $\operatorname{pg}(10)$ & This paper \\
\hline 2432705.716 & -579 & 2432705.635 & +0.081 & 1.0 & $\operatorname{pg}(21)$ & This paper \\
\hline 2433006.564 & -528 & 2433006.436 & +0.128 & 1.0 & $\operatorname{pg}(13)$ & This paper \\
\hline 2433507.869 & -443 & 2433507.771 & +0.098 & 1.0 & $\operatorname{pg}(9)$ & This paper \\
\hline 2434221.527 & -322 & 2434221.435 & +0.092 & 1.0 & $\operatorname{pg}(17)$ & This paper \\
\hline 2434887.978 & -209 & 2434887.915 & +0.063 & 3.0 & $\operatorname{pe}(7)$ & Walraven et al. (1958) \\
\hline 2435566.277 & -94 & 2435566.191 & +0.086 & 3.0 & pe(44) & Walraven et al. (1958) \\
\hline 2437005.435 & +150 & 2437005.316 & +0.119 & 3.0 & pe(11) & Irwin (1961) \\
\hline 2437648.231 & +259 & 2437648.204 & +0.027 & 3.0 & pe(5) & Mitchell et al. (1964) \\
\hline 2440780.086 & +790 & 2440780.070 & +0.016 & 3.0 & $\operatorname{pe}(31)$ & $\operatorname{Pel}(1976)^{a}$ \\
\hline 2444018.190 & +1339 & 2444018.101 & +0.089 & 2.0 & pe(5) & Harris (1980) \\
\hline 2444200.988 & +1370 & 2444200.941 & +0.047 & 2.0 & pe(8) & Caldwell et al. (2001) \\
\hline 2444666.950 & +1449 & 2444666.887 & +0.063 & 3.0 & pe(19) & Eggen (1985) \\
\hline 2447952.239 & +2006 & 2447952.102 & +0.137 & 3.0 & $\operatorname{IDD}(42)$ & ESA (1997) \\
\hline 2448123.280 & +2035 & 2448123.146 & +0.134 & 3.0 & $\operatorname{IDD}(37)$ & ESA (1997) \\
\hline 2448424.078 & +2086 & 2448423.947 & +0.131 & 3.0 & $\operatorname{IDD}(77)$ & ESA (1997) \\
\hline 2448689.538 & +2131 & 2448689.359 & +0.179 & 3.0 & IDD(49) & ESA (1997) \\
\hline 2449019.831 & +2187 & 2449019.650 & +0.181 & 3.0 & $\operatorname{IDD}(23)$ & ESA (1997) \\
\hline 2451644.531 & +2632 & 2451644.284 & +0.247 & 3.0 & $\operatorname{pe}(24)$ & Berdnikov \& Caldwell (2001) \\
\hline 2451963.024 & +2686 & 2451962.778 & +0.246 & 3.0 & pe(26) & Berdnikov \& Turner (2001) \\
\hline 2452358.255 & +2753 & 2452357.948 & +0.307 & 3.0 & pe(67) & Berdnikov \& Turner (2002) \\
\hline 2452647.252 & +2802 & 2452646.952 & +0.300 & 3.0 & pe(39) & Berdnikov \& Turner (2003) \\
\hline
\end{tabular}

${ }^{a}$ Photometry used as reference standard.

The resulting $\mathrm{O}-\mathrm{C}$ data for $\mathrm{T}$ Ant obtained by statistically adjusting each data set in phase and magnitude to the standard sequence are summarized in Table 1 and plotted in
Fig. 3. The symbol size in Fig. 3 is related to the weight assigned each O-C determination, as in the study of V1726 Cyg by Turner et al. (2001), which employed the scheme 


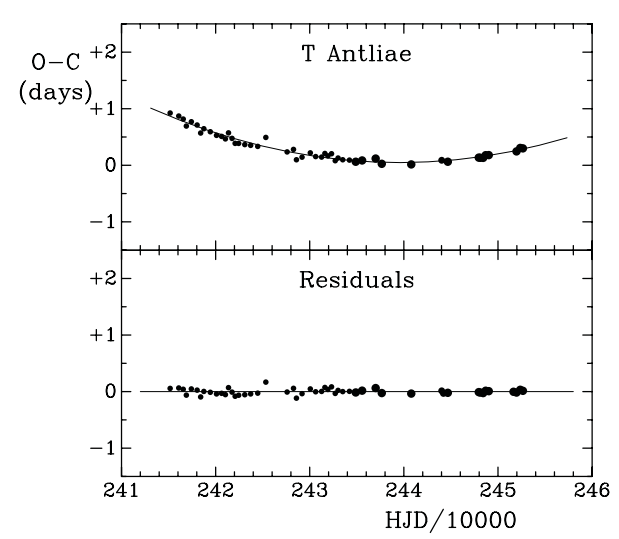

Fig. 3. O-C data for T Antliae plotted as a function of the observed Heliocentric Julian Date of light maximum (upper), and corrected for the gradual increase in pulsation period (lower). The size of the symbol increases with increasing weight for the data set.

established by Szabados (1977). All O-C estimates from photographic data were accorded a weight of 1.0, while O-C estimates derived from photoelectric observations were given a weight of 3.0 if the light curve coverage was thorough, otherwise a weight of 2.0. The $\mathrm{O}-\mathrm{C}$ data are closely approximated by a parabolic trend reflecting a regular period increase described by the relation:

$\mathrm{HJD}_{\max }=2436120.756+5.897915 E+4.80 \times 10^{-8} E^{2}$.

The inferred rate of period increase, $+0.514 \pm 0.016 \mathrm{~s} \mathrm{yr}^{-1}$, is similar to that found for other Cepheids established to be in the third crossing of the instability strip, as indicated in Fig. 4. Plotted in the diagram are similar data derived for a large selection of well-observed Cepheids that have reasonably wellestablished rates of period change (see Turner 1998; Berdnikov \& Ignatova 2000). The scientific study of evolutionary changes in pulsation period for Cepheids is still in its infancy, but the data from stellar evolutionary models currently indicate that the actual rate of period change for individual Cepheids depends primarily on three factors: (i) strip crossing mode, most Cepheids being in the second and third crossings, which are the longest (second crossings producing negative rates of period change, third crossings producing positive rates), (ii) location in the instability strip, with large amplitude Cepheids like $\mathrm{T}$ Ant tending to lie on the hot side of the strip (the lines in Fig. 4 represent theoretical expectations for Cepheids lying in the middle of the instability strip), and (iii) mode of pulsation, with overtone pulsators, presumably blue-edge Cepheids, undergoing period changes more typical of Cepheids of longer pulsation period. The result for T Ant, depicted by an open star symbol in Fig. 4, is consistent with values derived for a large set of classical Cepheids inferred to be crossing the instability strip for the third time during late stages of core helium burning. The third crossing is one of the slowest and accounts for most Cepheids with period increases (Turner 1998).

A statistical test was developed by Eddington \& Plakidis (1929) to establish the degree of regularity of pulsation in stars like T Ant, as illustrated by Percy et al. (1997), Percy \& Hale (1998), Percy \& Colivas (1999), and Turner et al. (2001). Such

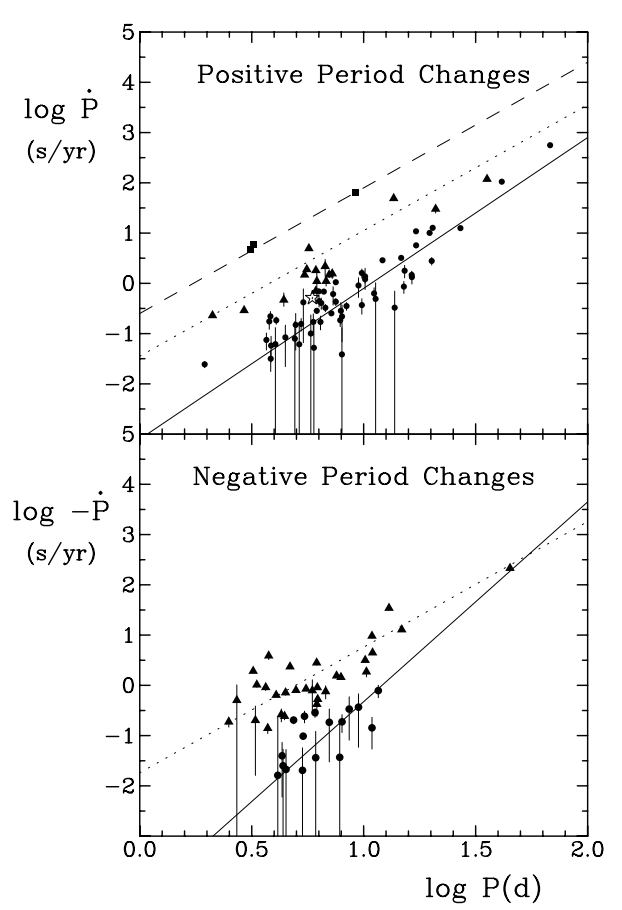

Fig. 4. Observed rates of period change (with calculated uncertainties) are compared with predictions from stellar evolutionary models and empirical calculations for stars lying in the center of the instability strip. From top to bottom the lines in the upper plot (period increases) correspond to first, fifth, and third crossings, while those in the lower plot (period decreases) correspond to fourth and second crossings. Different symbols are used to denote the assessment of likely strip crossing mode for individual Cepheids. T Antliae is represented by an open star symbol in the upper plot.

studies have revealed that most types of cool pulsators experience random fluctuations in pulsation period, but the test has not seen extensive use for Cepheids. A limited sample of results for classical Cepheids (see Turner \& Berdnikov 2001) indicates that chaotic fluctuations in pulsation period are less common and less marked in such stars than in longer period pulsators.

The test has been applied to T Ant (Turner \& Berdnikov 2001), and reveals no indication of random changes in pulsation period for the star. As practiced by Eddington \& Plakidis (1929), one examines time differences $a(r)$ for each of the $r$ th observed light maxima of the variable from the established ephemeris, and computes, without regard to sign, the accumulated delays $u(x)=a(r+x)-a(r)$ between maxima separated by $x$ cycles. The average value for all accumulated delays between light maxima separated by $x$ cycles, denoted $\langle u(x)\rangle$, is correlated with random fluctuations in period, $e$, by:

$<u(x)>^{2}=2 a^{2}+x e^{2}$,

where $a$ represents the magnitude of random errors in the measured times of light maxima. The results for $\mathrm{T}$ Ant are illustrated in Fig. 5 along with comparable data for $\delta$ Cep and SV Vul, the former displaying no evidence for chaotic behavior in its pulsation and the latter being recognized for a small degree of random period changes. A weighted least squares fit to the $\left\langle u(x)>^{2}\right.$ data for T Ant gives:

$<u(x)>^{2}=0.004( \pm 0.092)+0.000( \pm 0.001) x$, 


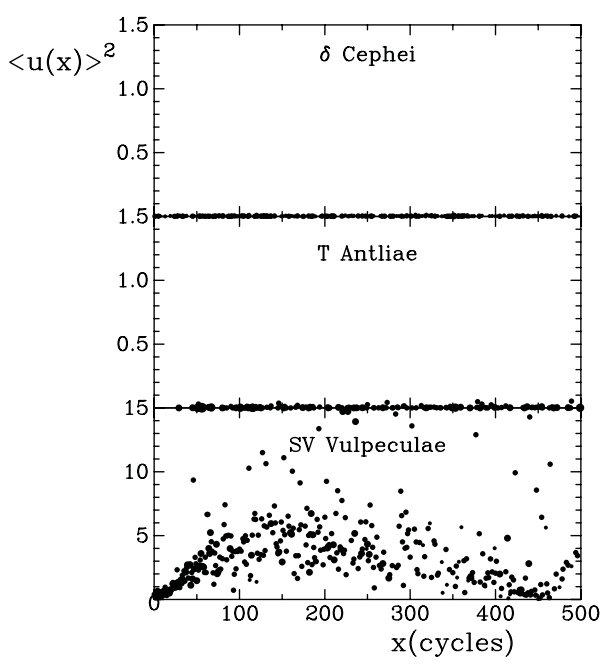

Fig. 5. $\mathrm{A}<u(x)>$ graph (plot of $<u(x)\rangle^{2}$ as a function of increasing cycle count difference $x$ ) derived from the residuals in the $\mathrm{O}-\mathrm{C}$ data for $\delta$ Cep (upper), T Ant (middle), and SV Vul (lower). The size of the symbol increases with increasing weight for the combined data pairs.

where the null value for the slope of the relation is consistent with no random cycle-to-cycle fluctuations in period for T Ant. The small amount of scatter evident in the data points of Fig. 3 does not arise from chaotic fluctuations in the star's pulsation period.

The residuals from the best fit to the $\mathrm{O}-\mathrm{C}$ data, although not indicative of random fluctuations in period, do display a marginally detectable sinusoidal trend that might be indicative of light travel time effects in the data arising from orbital motion (see lower part of Fig. 3). Unfortunately, the evidence rests entirely in data from the Harvard plates and may simply be spurious. Light travel time effects are typically very difficult to detect in $\mathrm{O}-\mathrm{C}$ data. If the trend is real, it would imply that $\mathrm{T}$ Ant is a binary system, which is an important possibility in view of the usefulness of Cepheid binaries for testing the parameters of pulsating stars. The inferred parameters for the orbit of the system ( $P \simeq 42.4$ years, $\left.a_{1} \sin i \simeq 10.8 \mathrm{AU}\right)$ generate a reasonable result for the mass of the Cepheid $\left(M_{1} \geq 0.7 M_{\odot}\right)$, given the obvious uncertainty in orbital inclination. It may therefore be practical to search for other evidence of possible binarity in T Ant that might be revealed for example by temporal variations in the systemic velocity of the system or by excess ultraviolet light.

Comments appended to $\mathrm{T}$ Ant in the recent edition of the General Catalogue of Variable Stars (Kholopov et al. 1985) imply some uncertainty regarding the population type of the Cepheid. Existing evidence based upon the inferred metallicity of T Ant (Harris 1981), a Baade-Wesselink radius typical of a Population I star (Laney 1995), the regular nature of its light curve (Figs. 1 and 2), and its observed rate of period change are all consistent with a classical Cepheid belonging to Population I rather than a type II Cepheid. The star's Galactic latitude of $11^{\circ}$ is the sole argument favoring thick disk or halo membership, but that can now be countered by the detection of other early-type stars in the field of T Ant.

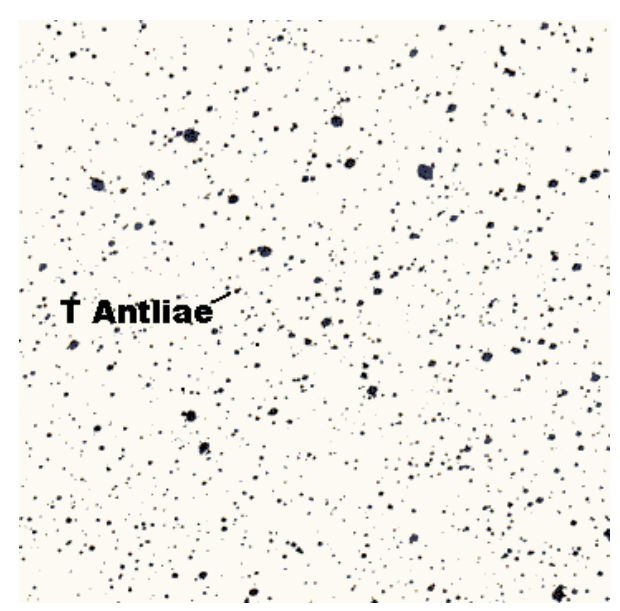

Fig. 6. The 3.5-diameter field near $\mathrm{T}$ Antliae centered on the putative open cluster identified in the text. North is up and east is to the left. Reproduced from the Photographischer Stern-Atlas Falkauer Atlas Südhimmel Zwischen $14^{\circ}$ Südlicher Deklination und Südpol (Vehrenberg 1964).

\section{A cluster near T Antliae}

After the detection of period changes in T Ant, an inspection was made of the field of the Cepheid on the ESO Sky Survey. One of us (D.G.T.) has experience recognizing poorly populated open clusters on survey images from studies of many of the Cepheid calibrating clusters and from new detections of such clusters (Turner 1984, 1985; Turner et al. 1993, 1997, 1998). Our inspection revealed a slight enhancement in star density near $\mathrm{T}$ Ant similar to that of a poorly populated open cluster, an impression strengthened when the Harvard patrol plates of the field were examined. The putative cluster is uncatalogued, but is centered near $\alpha_{1950}=09^{\mathrm{h}} 29^{\mathrm{m}}$, $\delta_{1950}=-36^{\circ} 24^{\prime}$, which translates into an IAU designation of C0939-364 if its existence is confirmed.

The star density enhancement indicative of the cluster shows up in deep images of the Harvard patrol series, but less noticeably on the Digitized Sky Survey at faint magnitudes. It is also noticeable to a moderate extent on small scale views of the field, as indicated by Fig. 6, which illustrates the 3.5 field centered on the putative cluster as it appears in the Vehrenberg Atlas (Vehrenberg 1964). The Cepheid T Ant sits on the eastern edge of the cluster.

The sparse nature of the cluster suggests that star counts of the field would provide only marginal confirmation of the density enhancement of moderately bright stars that is visible to the eye. We therefore anticipated that spectroscopic information might be of greater value in assessing the cluster's reality, and initiated a search of the Harvard Plate Collection for objective-prism plates of the field near T Ant. The Harvard Collection includes the objective prism plates used by Annie Jump Cannon for the classification of stars in the Henry Draper Catalogue, but we found that those plates were not deep enough to add to the HD spectral types already available. A further search yielded a $90^{\mathrm{m}}$ objective prism exposure obtained from Harvard's Boyden Station in Bloemfontein, South Africa, on the evening of February 7/8, 1946, that contained low 


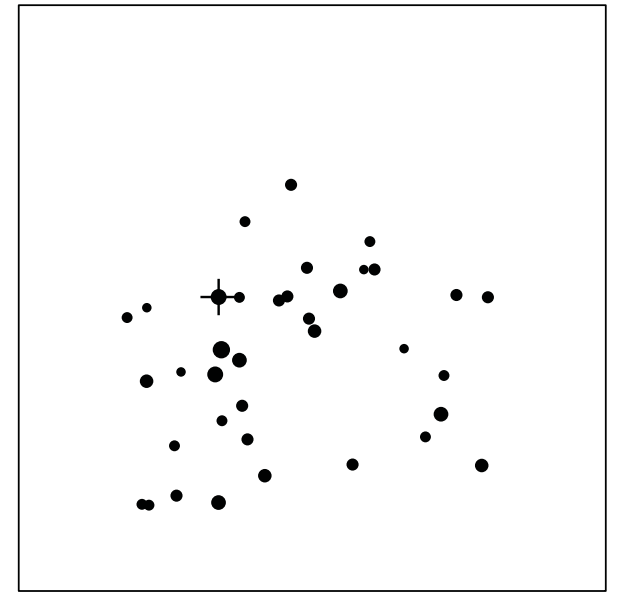

Fig. 7. The field of Fig. 5 showing the distribution of potential members of the T Ant cluster identified here. Symbol size is proportional to star brightness, and the cross indicates the position of $\mathrm{T}$ Ant.

dispersion spectra of stars near $\mathrm{T}$ Ant to about 12th magnitude photographic. From inspection of the plate we were able to identify numerous B-type and A-type stars in the field of the Cepheid.

Because of the low dispersion of the objective-prism spectra (about $350 \AA \mathrm{mm}^{-1}$ at $\mathrm{H} \gamma$ ), it was not possible to assign temperature subtypes or luminosity classes to the stars. B-type stars were identified from spectra displaying the Balmer lines of hydrogen but no ionized calcium lines (e.g., Ca II K). A-type stars were identified from spectra displaying the hydrogen Balmer lines and the K-line.

A significant fraction of the stars in the field of $\mathrm{T}$ Ant prove to be of B-type or early A-type, but many seem to lie foreground to the Cepheid. The latter are generally bright, blue ( $B-V \leq 0.13$ ), and of small interstellar reddening, consistent with the small reddenings of $E_{B-V} \leq 0.05$ observed for stars of $d \leq 1 \mathrm{kpc}$ in adjacent fields lying closer to the Galactic plane (Neckel \& Klare 1980; Slawson \& Reed 1988). In those fields the reddening increases beyond distances of $\sim 1 \mathrm{kpc}$, so we assume that the more heavily reddened B-type stars near T Ant are at least that distant. Candidate cluster stars, including what may be two K supergiant members, are identified in Table 2 along with obvious foreground stars (stars of small interstellar reddening), where the cited co-ordinates and photometric data are taken where possible from the Hipparcos/Tycho catalogue. The spatial distribution of stars potentially related to $\mathrm{T}$ Ant is illustrated in Fig. 7.

The identification of the two K-type stars as potential cluster members is highly uncertain, and rests mainly on their close proximity to $\mathrm{T}$ Ant and their similar apparent brightness to the Cepheid. For star 1 the identification is supported by the star's inferred reddening, but in both cases higher dispersion spectroscopic observations would help decide the case. $\mathrm{K}$ giants are a common component of the Galactic disk.

The reddening near T Ant is fairly small, given that a cluster of galaxies is visible on Sky Survey images of the eastern portion of the field. Four, bright, early-type stars near T Ant have MK spectral types (B8 II, A0 V, A0 V, and A1 V), and their derived mean reddening of $E_{B-V}=0.05 \pm 0.01$ confirms their foreground status and is consistent with the foreground reddening typical of nearby Galactic fields (Neckel \& Klare 1980; Slawson \& Reed 1988). The reddening near T Ant must also be relatively smooth, given the lack of any obvious patchiness in star densities, which is typical of fields containing differential reddening.

A larger space reddening applies to $\mathrm{T}$ Ant and potentially associated stars identified in Table 2, which presumably lie beyond the dust complex at about $1 \mathrm{kpc}$ distance. Curiously, the data for several stars, including the apparently anomalous star 7, which has the $B-V$ color of a late-type star, appear to be too red for B spectral types, especially given the apparent absence of patchy obscuration noted above. Further discussion of the anomaly is given later.

Estimates for the reddening of $\mathrm{T}$ Ant from Walraven photometry (Pel 1978) and from its mean spectral type are given in the top portion of Table 3 , and imply a reddening near $E_{B-V} \simeq 0.3$ for the field. Confirmation of such a result would normally present problems, since only broad band $B-V$ colors and low quality spectral types are available. The problem was addressed by examining in the lower portion of Table 3 the reddening of specific potential companion stars to $\mathrm{T}$ Ant: star 1, which has a HD type of $\mathrm{K} 2$ and is assumed here to be a class Ib or II supergiant member of the group; five stars of about 10th magnitude, two with HD types of A0, assumed to be either B9.5 or A0 giant stars lying near the tip of the evolved cluster main sequence; and eight faint stars assumed to lie on the evolved portion of the cluster main sequence, for which an intrinsic color was adopted from the pulsation period of T Ant (see Turner 1996). Intrinsic colors for the possible K supergiant and T Ant were adopted from Fernie (1963), Johnson (1966), FitzGerald (1970), Kron (1978), and SchmidtKaler (1982), and all results were transformed to the equivalent reddening for a $\mathrm{B} 0$ star.

There is an obvious danger in such a procedure since it appears to assume the expected result, namely that there is a star cluster associated with $\mathrm{T}$ Ant. Yet the results are relatively unaltered without such an assumption, since the lack of detectable $\mathrm{Ca}$ II $\mathrm{K}$ lines in the B-type stars leads to almost identical color excesses. From Table 3 we find a mean reddening for potential group stars (other than $\mathrm{T}$ Ant) of $E_{B-V}(\mathrm{~B} 0)=$ $0.316 \pm 0.014$ s.d., which corresponds to a field reddening for T Ant of $E_{B-V}($ Cepheid $)=0.30 \pm 0.01$.

The spatial distribution of B-type stars near T Ant can also be established from the data plotted in Fig. 7. In general there is a decrease in B-star densities, as expected, as one views towards higher Galactic latitudes, yet there is also a slight clustering towards the putative cluster identified by eye. The densities of B-type stars (B7-A0) in the field to various limiting magnitudes are about $40 \%$ of the values found in a nearby region of the Galactic plane in Vela studied by Reed \& Slawson (1990), which is typical of what one expects for the decrease in star densities as one views away from the Galactic plane. The B-star densities increase to $50 \%$ of the values in the Galactic plane between $V=11$ and 12, however, whereas a decrease is expected given that fainter stars are also more distant stars, which should become less common as one views further from 
Table 2. Potential companion stars near T Antliae.

\begin{tabular}{|c|c|c|c|c|c|c|}
\hline Star & Identification & $\alpha_{2000}$ & $\delta_{2000}$ & Sp.T. & $V$ & $B-V$ \\
\hline \multicolumn{7}{|c|}{ Potential Cluster Stars } \\
\hline 1 & HD 82872 & 09:33:46 & $-36: 55.9$ & $\mathrm{~K} 2(\mathrm{Ib} ?)$ & 8.92 & 1.602 \\
\hline 2 & $\mathrm{CD}-36^{\circ} 5777$ & 09:33:57 & $-37: 04.7$ & $\mathrm{~K}(\mathrm{I} ?)$ & 9.20 & 1.489 \\
\hline 3 & T Ant & 09:33:51 & $-36: 36.9$ & F6 Iab & 9.34 & 0.720 \\
\hline 4 & $\mathrm{CD}-36^{\circ} 5680$ & 09:27:14 & $-37: 19.0$ & B & 9.76 & 0.320 \\
\hline 5 & $\mathrm{CD}-37^{\circ} 5876$ & 09:33:51 & $-37: 50.6$ & B & 9.77 & 0.210 \\
\hline 6 & HD 82784 & 09:33:14 & $-36: 59.6$ & $\mathrm{~A} 0$ & 9.85 & 0.280 \\
\hline 7 & Ty7166 18091 & 09:30:14 & $-36: 34.8$ & $\mathrm{~B}$ & 9.94 & 0.958 \\
\hline 8 & Ty7166 29841 & 09:31:00 & $-36: 49.2$ & $\mathrm{~B}$ & 10.01 & 0.280 \\
\hline 9 & Тy7691 43741 & 09:26:02 & $-37: 37.3$ & $\mathrm{~B}$ & 10.21 & 0.281 \\
\hline 10 & $\mathrm{CD}-36^{\circ} 5805$ & 09:35:59 & $-37: 07.1$ & $\mathrm{~B}$ & 10.41 & 0.247 \\
\hline 11 & Тy7692 30741 & 09:32:28 & $-37: 41.0$ & $\mathrm{~B}$ & 10.44 & 0.297 \\
\hline 12 & Тy7166 11291 & 09:32:03 & $-36: 38.2$ & $\mathrm{~B}$ & 10.53 & 0.253 \\
\hline 13 & Ty7166 29761 & 09:33:09 & $-37: 15.9$ & B & 10.53 & 0.380 \\
\hline 14 & Тy7691 43141 & 09:29:52 & $-37: 37.0$ & $\mathrm{~B}$ & 10.55 & 0.236 \\
\hline 15 & Тy7166 10661 & 09:31:42 & $-35: 56.7$ & $\mathrm{~B}$ & 10.64 & 0.162 \\
\hline 16 & Тy7166 11211 & 09:31:10 & $-36: 44.7$ & B & 10.67 & 0.237 \\
\hline 17 & Тy7166 11951 & 09:31:48 & $-36: 36.8$ & $\mathrm{~B}$ & 10.69 & 0.236 \\
\hline 18 & Тy7165 12941 & 09:26:47 & $-36: 36.2$ & $\mathrm{~B}$ & 10.78 & 0.227 \\
\hline 19 & Тy7166 7651 & 09:29:13 & $-36: 27.1$ & $\mathrm{~B}$ & 10.79 & 0.435 \\
\hline 20 & Тy7166 2941 & 09:33:00 & $-37: 28.0$ & $\mathrm{~B}$ & 10.84 & 0.399 \\
\hline 21 & Ty7165 17531 & 09:25:51 & $-36: 37.0$ & $\mathrm{~B}$ & 10.88 & 0.172 \\
\hline 22 & Ty7692 5601 & 09:35:06 & $-37: 48.2$ & B & 10.92 & 0.359 \\
\hline 23 & Тy7166 10091 & 09:31:13 & $-36: 26.5$ & $\mathrm{~B}$ & 10.98 & 0.406 \\
\hline 24 & Ty7692 8281 & 09:35:55 & $-37: 51.6$ & $\mathrm{~B}$ & 11.04 & 0.290 \\
\hline 25 & Ty7166 3521 & $09: 27: 42$ & $-37: 27.1$ & B & 11.10 & 0.244 \\
\hline 26 & Ty7692 29141 & 09:36:08 & $-37: 51.3$ & $\mathrm{~B}$ & 11.14 & 0.354 \\
\hline 27 & Тy7166 2871 & 09:27:09 & $-37: 05.1$ & $\mathrm{~B}$ & 11.15 & 0.167 \\
\hline 28 & Ty7166 7371 & 09:33:45 & $-37: 21.3$ & $\mathrm{~B}$ & 11.15 & 0.509 \\
\hline 29 & Тy7166 20361 & 09:29:21 & $-36: 17.1$ & $\mathrm{~B}$ & 11.16 & 0.175 \\
\hline 30 & Тy7692 1391 & 09:35:10 & $-37: 30.3$ & $\mathrm{~B}$ ? & 11.32 & 0.145 \\
\hline 31 & Ty7179 24031 & $09: 36: 34$ & $-36: 44.3$ & $?$ & 11.40 & 0.149 \\
\hline 32 & $\cdots$ & 09:33:14 & $-36: 37.1$ & B & $11.4:$ & $\cdots$ \\
\hline 33 & Ty7166 14361 & 09:33:04 & $-36: 09.9$ & $\mathrm{~B}$ & 11.47 & 0.169 \\
\hline 34 & Тy7166 13311 & 09:28:20 & $-36: 55.5$ & $\mathrm{~B}$ & 11.52 & 0.201 \\
\hline 35 & Тy7166 18151 & 09:29:32 & $-36: 27.1$ & $\mathrm{~B}$ & 11.59 & 0.177 \\
\hline 36 & $\ldots$ & 09:35:59 & $-36: 40.8$ & B & 11.6: & $\ldots$ \\
\hline \multirow[t]{2}{*}{37} & $\cdots$ & 09:34:58 & $-37: 03.8$ & B & $11.8:$ & $\cdots$ \\
\hline & Foreground Stars & & & & & \\
\hline 38 & HD 81889 & $09: 27: 23$ & $-36: 52.8$ & $\mathrm{~A} 1 \mathrm{~m}$ & 7.81 & 0.275 \\
\hline 39 & HD 82317 & 09:30:15 & $-37: 05.3$ & $\mathrm{~A} 0$ & 8.08 & 0.104 \\
\hline 40 & HD 83011 & 09:34:28 & $-37: 25.3$ & $\mathrm{~A} 0$ & 8.34 & 0.017 \\
\hline 41 & HD 81800 & 09:26:51 & $-36: 00.2$ & B9 & 8.58 & 0.037 \\
\hline 42 & HD 82097 & 09:28:47 & $-36: 56.1$ & A1m & 8.80 & 0.322 \\
\hline 43 & HD 82344 & 09:30:17 & $-36: 34.8$ & $\mathrm{~A} 0$ & 8.95 & 0.029 \\
\hline 44 & HD 81633 & $09: 25: 47$ & $-37: 35.9$ & $\mathrm{~A} 0$ & 9.16 & 0.033 \\
\hline 45 & HD 82562 & 09:31:49 & $-36: 32.3$ & A0 & 9.26 & 0.034 \\
\hline 46 & HD 81778 & 09:26:39 & $-36: 50.5$ & B9 & 9.60 & -0.007 \\
\hline 47 & HD 82596 & 09:32:00 & $-37: 02.6$ & $\mathrm{~A} 2$ & 9.90 & 0.066 \\
\hline 48 & HD 82343 & 09:30:19 & $-36: 30.2$ & A0 & 10.00 & 0.110 \\
\hline 49 & Ty7166 26771 & 09:30:14 & $-36: 55.1$ & $\mathrm{~B}$ & 10.17 & 0.115 \\
\hline 50 & HD 817161 & 09:26:18 & $-36: 12.5$ & $\mathrm{~A} 0$ & 10.18 & 0.046 \\
\hline 51 & Ty7691 43221 & 09:26:32 & $-37: 38.0$ & B & 10.23 & 0.127 \\
\hline 52 & $\mathrm{CD}-37^{\circ} 5865$ & 09:33:07 & $-37: 34.1$ & B & 10.49 & 0.068 \\
\hline
\end{tabular}


Table 3. Estimated reddening of $\mathrm{T}$ Antliae cluster.

\begin{tabular}{|c|c|c|c|c|c|}
\hline Star & $B-V$ & $(B-V)_{0}$ & $E_{B-V}$ & $E_{B-V}(\mathrm{~B} 0)$ & Sp.T. Adopted \\
\hline \multirow[t]{2}{*}{ T Ant } & +0.72 & . & 0.25 & 0.27 & Pel (1978) \\
\hline & +0.72 & +0.46 & 0.26 & 0.28 & F6 Iab \\
\hline 1 & +1.61 & +1.32 & 0.29 & 0.33 & $\mathrm{~K} 2(\mathrm{Ib})$ \\
\hline 4 & +0.32 & +0.00 & 0.32 & 0.33 & A0 (adopted) \\
\hline 6 & +0.28 & +0.00 & 0.28 & 0.29 & A0 \\
\hline 8 & +0.28 & -0.03 & 0.31 & 0.32 & B9.5 (adopted) \\
\hline 9 & +0.28 & -0.03 & 0.31 & 0.32 & B9.5 (adopted) \\
\hline 11 & +0.30 & -0.03 & 0.33 & 0.34 & B9.5 (adopted) \\
\hline 15 & +0.16 & -0.14 & 0.30 & 0.31 & B6 (turnoff) \\
\hline 21 & +0.17 & -0.14 & 0.31 & 0.32 & B6 (turnoff) \\
\hline 27 & +0.17 & -0.14 & 0.31 & 0.32 & B6 (turnoff) \\
\hline 29 & +0.18 & -0.14 & 0.32 & 0.32 & B6 (turnoff) \\
\hline 30 & +0.15 & -0.14 & 0.29 & 0.29 & B6 (turnoff) \\
\hline 31 & +0.15 & -0.14 & 0.29 & 0.30 & B6 (turnoff) \\
\hline 33 & +0.17 & -0.14 & 0.31 & 0.32 & B6 (turnoff) \\
\hline 35 & +0.18 & -0.14 & 0.32 & 0.33 & B6 (turnoff) \\
\hline Average & & & & $\begin{array}{c}0.32 \\
\pm 0.01 \text { s.d. }\end{array}$ & \\
\hline
\end{tabular}

the main plane of the Galaxy. In Vela there is also a significant increase in extinction beyond distances of $1 \mathrm{kpc}$ and the line of sight intersects at least three OB associations (Slawson \& Reed 1988), so the lack of a noticeable decrease in B-star densities at faint magnitudes towards $\mathrm{T}$ Ant implies that the line of sight must contain one or more distant stellar groups. A distant group of B-type stars in the field of the Cepheid could provide most of the additional B-type stars detected in the present survey.

\section{Is T Antliae a cluster member?}

The expected luminosity for $\mathrm{T}$ Ant as a classical Cepheid with a pulsation period of 5.898 is $\left\langle M_{V}\right\rangle=-3.42 \pm 0.07$ (Turner 1992), which leads to an expected distance modulus of $V_{0}-M_{V}=11.81 \pm 0.07$ (for an assumed value of $R=3.0$ ), corresponding to a distance of $2.30 \pm 0.07 \mathrm{kpc}$. A distanceadjusted color-magnitude diagram for the putative cluster corrected for an assumed constant foreground reddening is plotted in the upper middle portion of Fig. 8, with similar data for the open clusters NGC 6649 and Roslund 3 plotted for comparison purposes in the upper portion and lower middle portions of the figure, respectively. The double-mode Cepheid V367 Sct is a member of NGC 6649 (e.g., Turner 1981), and also has a period for fundamental mode pulsation very similar to that of T Ant, so its surrounding cluster provides a suitable comparison sequence for the stars near T Ant.

It is apparent from Fig. 8 that stars identified here as being potentially associated with $\mathrm{T}$ Ant share some similarities to stars on the evolved main sequence of NGC 6649. The latter are a good match to theoretical isochrones for $\log t=7.9 \pm 0.05$, which corresponds to an age of $(79 \pm 9) \times 10^{6}$ years. In the case of stars associated with $\mathrm{T}$ Ant, the bluest are a reasonable match to theoretical isochrones for $\log t=8.0 \pm 0.05$, which corresponds to an age of $(100 \pm 12) \times 10^{6}$ years. The slightly

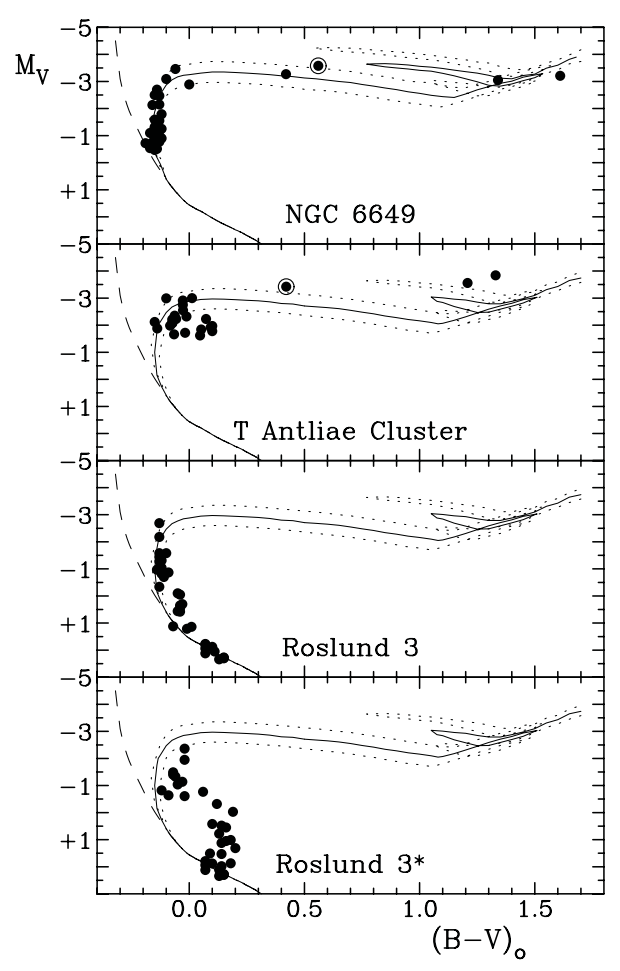

Fig. 8. Reddening and distance-adjusted magnitudes and colors for stars in NGC 6649 (upper), the T Antliae cluster (upper middle), Roslund 3 (lower middle), and Roslund 3 as corrected only for foreground reddening (lower) plotted relative to the zero-age main sequence (ZAMS) relation (dashed line) corresponding to distances of $1.65,2.30 \mathrm{kpc}$, and $2.29 \mathrm{kpc}$ (two lower plots) respectively. Theoretical isochrones are shown for evolved stars with ages of $\log t=$ 7.8, 7.9, and 8.0 (dotted, solid, and dotted lines, respectively) in the upper diagram, and $\log t=7.9,8.0$, and 8.1 (dotted, solid, and dotted lines, respectively) in the lower three diagrams. The data for V367 Sct (in NGC 6649) and T Ant are represented by circled points.

older implied age for T Ant is consistent with its shorter pulsation period relative to V367 Sct (5.898 versus 6.293). T Ant is also bluer than V367 Sct, which is a double-mode Cepheid, and the two stars identified as possible $\mathrm{K}$ supergiants are bluer than the plotted isochrones. The latter might be the result of a metallicity for group stars that is less than the solar value, while the former is consistent with the evidence from the star's large light amplitude and relatively rapid rate of period change for a third crosser, both of which argue that $\mathrm{T}$ Ant lies on the blue side of the instability strip.

The inordinately large scatter in the colors of stars inferred to lie on the main-sequence turnoff for the T Ant group is curious, but cannot be attributed to a larger space reddening for the stars since there is no evidence to indicate the existence of either clumpy dust in the field or dust clouds lying beyond those roughly $1 \mathrm{kpc}$ distant. Even if the affected objects are field stars rather than group members, the same problem arises. Their colors are simply too red for stars identified spectroscopically as B-type, even when corrected for a field reddening of $E_{B-V}=0.32$. A larger reddening for the stars would be inconsistent with the run of extinction with distance in this direction. Either the $B-V$ data for stars in the Hipparcos/Tycho database are erroneous or the stars must exhibit excess 
reddening, perhaps arising from circumstellar extinction, as found for rapidly rotating main-sequence members of Roslund 3 by Turner (1993), for example.

The color-magnitude diagram for Roslund 3 plotted in Fig. 8 indicates that the cluster is very similar in age to the $\mathrm{T}$ Antliae group. The deleterious effects of circumstellar reddening on the colors of member stars is revealed in the lower part of Fig. 8, in which the colors of cluster stars have been corrected only for foreground reddening but not circumstellar reddening. The redward spread from the evolved portion of the cluster main sequence is similar to what is found for objects in the T Antliae group, where individual reddenings cannot be derived. A smaller "reddening" effect is also mimicked by rapidly rotating stars viewed nearly equator-on, as seen in the reddening-corrected data for Roslund 3 stars. It is possible that a similar effect arising from circumstellar reddening affects stars in the T Antliae cluster. High quality spectroscopic and photometric observations, and two color photometry in particular, are essential for testing that possibility. There is some evidence to suggest that such an effect is encountered fairly frequently in clusters with ages similar to those of Cepheid variables (Turner 1991). It is not seen in the data for NGC 6649 simply because the colors for cluster stars have already been corrected for differential reddening.

A proper distance estimate for the pseudo-cluster requires a deep photometric study of the field that reaches stars lying on the zero-age main sequence. Such stars are presumably fainter than $V=13$ or 14 , which is within the reach of modest-sized telescopes. An observational program to obtain deep photometry of the field and medium dispersion spectroscopy of bright group members is needed to confirm our suspicions that $\mathrm{T}$ Ant is a cluster member, and therefore a potential calibrating object for the Cepheid period-luminosity relation.

Since the main data for potential group stars were obtained from the Hipparcos/Tycho catalogue (ESA 1997), there are parallax and proper motion data available for consideration, although many of the values are of low quality. The proper motions, in particular, are small and dominated by the solar motion, so they are of limited use for testing the reality of the cluster. The weighted mean parallax for 34 cluster stars, including $\mathrm{T}$ Ant, with weights assigned according to the cited uncertainties in the individual parallaxes, is $\pi=0.425 \pm 0.804$ mas, which corresponds to a distance of $2.15_{-1.54}^{+\infty} \mathrm{kpc}$. The weighted mean parallax for group stars is clearly too uncertain to constrain their distance, although it does confirm that they are not nearby objects, as we have already concluded. Further study of the group is still essential for verifying its existence.

\section{Discussion}

The group of stars identified here as being potentially associated with $\mathrm{T}$ Ant is referred to as a cluster, but the dispersion of the B-type stars across the field of Fig. 6 amounts to about 2:8, or $112 \mathrm{pc}$ at the inferred distance, which is more typical of an association or dissolved cluster. The innermost group of stars identified with the density enhancement in Fig. 5 has a diameter of $23^{\prime}$, or $15 \mathrm{pc}$, which is more typical of a large cluster. Inspection of the field of $\mathrm{T}$ Ant does reveal a few, smaller, compact, anonymous star clusters that may also be related to $\mathrm{T}$ Ant, but at much larger projected distances from $\mathrm{T}$ Ant than the stars of Table 2. The B-type stars identified here therefore constitute what may be the remains of an open cluster that is now dissolving into the field. The Orion Ring surrounding $\epsilon$ Orionis in the Belt of Orion is an example of a star cluster in an earlier stage of dissolution, while the smattering of stars associated with the Cepheid SU Cyg (Turner et al. 1998) constitutes what appears to be a cluster in a more advanced state of dissolution. Such "cluster remains" may be typical of many of the star groups associated with Cepheids, which are fairly well advanced in an evolutionary sense.

Our purpose here is to draw attention to the possible association of the Cepheid T Ant with B-type stars, and two potential $\mathrm{K}$ supergiants, in its immediate surrounding. Information about the Cepheid and its putative cluster has been gleaned so far from archival data, which present a reasonable case for potential cluster membership. The next logical step is to initiate an observational program to test the arguments advanced here for cluster membership of T Ant.

Acknowledgements. This investigation was supported by research funding awarded through the Small Research Grants Program of the American Astronomical Society (AAS), in part by funding from the Cecelia Payne and Sergei Gaposchkin Memorial Fund administered by the AAS, and through the Russian Foundation of Basic Research and the State Science and Technology Program "Astronomy" to LNB. We are grateful to Alison Doane and the Director of the Harvard College Observatory for the use of the photographic plate collection, which generated a large portion of the data used for this study.

\section{References}

Berdnikov, L. N. 1992, Sov. Astron. Lett., 18, 207

Berdnikov, L. N. 1994, Astron. Lett., 20, 232

Berdnikov, L. N. 1995, in Astrophysical Applications of Stellar Pulsation, IAU Colloq. 155, ed. R. S. Stobie, \& P. A. Whitelock, ASP Conf. Ser., 83, 349

Berdnikov, L. N. 2002, unpublished data base available electronically Berdnikov, L. N., \& Caldwell, J. A. R. 2001, J. Astron. Data, 7, No. 3 Berdnikov, L. N., \& Ignatova, V. V. 2000, in The Impact of LargeScale Surveys on Pulsating Star Research, IAU Colloq. 176, ed. L. Szabados, \& D. W. Kurtz, ASP Conf. Ser., 203, 244

Berdnikov, L. N., \& Pastukhova, E. N. 1995, Astron. Lett., 21, 369

Berdnikov, L. N., \& Turner D. G. 2001, ApJS, 137, 209

Berdnikov, L. N., \& Turner, D. G. 2002, ApJS, submitted

Berdnikov, L. N., \& Turner D. G. 2003, unpublished

Berdnikov, L. N., Ignatova, V. V., Pastukhova, E. N., \& Turner, D. G. 1997, Astron. Lett., 23, 177

Berdnikov, L. N., Ignatova, V. V., Caldwell, J. A. R., \& Koen, C. 2000, New Astron., 4, 625

Caldwell, J. A. R., Coulson, I. M., Dean, J. F., \& Berdnikov, L. N. 2001, J. Astron. Data, 7, No. 4

Croswell, K. 2002, S\&T, July, 38

Eddington, A. S., \& Plakidis, S. 1929, MNRAS, 90, 65

Eggen, O. J. 1985, AJ, 90, 1297

ESA 1997, The Hipparcos and Tycho Catalogues, ESA SP-1200

Fernie, J. D. 1963, AJ, 68, 780

FitzGerald, M. P. 1970, A\&A, 4, 234

Harris, H. C. 1980, Ph.D. Thesis, University of Washington

Harris, H. C. 1981, AJ, 86, 719 
Irwin, J. B. 1961, ApJS, 6, 253

Johnson, H. L. 1966, ARA\&A, 4, 193

Kholopov, P. N., Samus, N. N., Frolov, M. S., et al. 1985, General Catalogue of Variable Stars, Fourth edition (Moscow: Nauka Publ. House)

Laney, C. D. 1995, in Astrophysical Applications of Stellar Pulsation, IAU Colloq. 155, ed. R. S. Stobie, \& P. A. Whitelock, ASP Conf. Ser., 83, 367

Kron, G. E. 1978, AJ, 83, 1195

Mitchell, R. I., Iriarte, B., Steinmetz, D., \& Johnson, H. L. 1964, Bol. Obs. Tonantzintla y Tacubaya, 3,153

Neckel, Th., \& Klare, G. 1980, A\&AS, 42, 251

Pel, J. W. 1976, A\&AS, 24,413

Pel, J. W. 1978, A\&A, 62, 75

Percy, J. R., \& Hale, J. 1998, PASP, 110, 1428

Percy, J. R., \& Colivas, T. 1999, PASP, 111, 94

Percy, J. R., Bezuhly, M., Milanowski, M., \& Zsoldos, E. 1997, PASP, 109,264

Reed, B. C., \& Slawson, R. W. 1990, AJ, 100, 156

Schmidt-Kaler, Th. 1982, in Landolt- Börnstein, Numerical Data and Functional Relationships in Science and Technology, Group VI, vol. 2b, ed. K Schaifers, \& H. H. Voigt (Berlin: Springer), Sect. 4

Slawson, R. W., \& Reed, B. C. 1988, AJ, 96, 988

Szabados, L. 1977, Comm. Konkoly Obs. Hung. Acad. Sci., No. 70
Turner, D. G. 1981, AJ, 86, 231

Turner, D. G. 1984, PASP, 96, 422

Turner, D. G. 1985, JRASC, 79, 175

Turner, D. G. 1991, JRASC, 85, 216

Turner, D. G. 1992, AJ, 104, 1865

Turner, D. G. 1993, A\&AS, 97, 755

Turner, D. G. 1996, JRASC, 90, 82

Turner, D. G. 1998, J. AAVSO, 26, 101

Turner, D. G., \& Berdnikov, L. N. 2001, Odessa Astron. Publ., 14, 170

Turner, D. G., Mandushev, G. I., \& Welch, G. A. 1997, AJ, 113, 2104

Turner, D. G., Horsford, A. J., \& MacMillan, J. D. 1999, J. AAVSO, 27,5

Turner, D. G., Billings, G. W., \& Berdnikov, L. N. 2001, PASP, 113, 715

Turner, D. G., van den Bergh, S., Younger, P. F., Danks, T. A., \& Forbes, D. 1993, ApJS, 85, 119

Turner, D. G., Ibrahimov, M. A., Mandushev, G. I., Berdnikov, L. N., \& Horsford, A. J. 1998, JRASC, 92, 145

Vehrenberg, H. 1964, Photographischer Stern-Atlas Falkauer Atlas Südhimmel Zwischen $14^{\circ}$ Südlicher Deklination und Südpol (Düsseldorf: Treugesell-Verlag)

Walraven, T., Mueller, A. B., \& Oosterhoff, P. T. 1958, Bull. Astron. Inst. Netherl., 14, 81 